\title{
Disruption of the M949_RS01915 gene changed the bacterial lipopolysaccharide pattern, pathogenicity and gene expression of Riemerella anatipestifer
}

\author{
Yafeng Dou, Xiaolan Wang, Guijing Yu, Shaohui Wang, Mingxing Tian, Jingjing Qi, Tao Li, Chan Ding \\ and Shengqing $\mathrm{Yu}^{*}$
}

\begin{abstract}
Riemerella anatipestifer is an important pathogen that causes septicemia anserum exsudativa in ducks. Lipopolysaccharide (LPS) is considered to be a major virulence factor of $R$. anatipestifer. To identify genes involved in LPS biosynthesis, we screened a library of random Tn4351 transposon mutants using a monoclonal antibody against $R$. anatipestifer serotype 1 LPS (anti-LPS MAb). A mutant strain RA1067 which lost the reactivity in an indirect ELISA was obtained. Southern blot and sequencing analyses indicated a single Tn4351 was inserted at $116 \mathrm{bp}$ in the M949_ RS01915 gene in the RA1067 chromosomal DNA. Silver staining and Western blot analyses indicated that the RA1067 LPS was defected compared to the wild-type strain CH3 LPS. The RA1067 displayed a significant decreased growth rate at the late stage of growth in TSB in comparison with $\mathrm{CH} 3$. In addition, RA1067 showed higher susceptibility to complement-dependent killing, more than 360-fold attenuated virulence based on the median lethal dose determination, increased bacterial adhesion and invasion capacities to Vero cells and significantly decreased blood bacterial loads in RA1067 infected ducks, when compared to the CH3. An animal experiment indicated that inactivated RA1067 cells was effective in cross-protecting of the ducks from challenging with R. anatipestifer strains WJ4 (serotype 1), Yb2 (serotype 2) and HXb2 (serotype 10), further confirming the alteration of the RA1067 antigenicity. Moreover, RNASeq analysis and real-time PCR verified two up-regulated and three down-regulated genes in RA1067. Our findings demonstrate that the M949_RS01915 gene is associated to bacterial antigenicity, pathogenicity and gene regulation of $R$. anatipestifer.
\end{abstract}

\section{Introduction}

Riemerella anatipestifer is a Gram-negative, non-motile, non-spore-forming, rod-shaped bacterium, which causes epizootic infectious disease in poultry, especially in ducks [1-3]. R. anatipestifer infected ducks were characterized by airsacculitis, pericarditis, perihepatitis, diarrhoea, ataxia, meningitis and depression of growth rate [4]. Until now, 21 serotypes of $R$. anatipestifer have been identified, there is poor cross-protection among them [5-7]. The occurrence of different serotypes has been

\footnotetext{
*Correspondence: yus@shvri.ac.cn

Shanghai Veterinary Research Institute, Chinese Academy of Agricultural Sciences, Shanghai 200241, China
}

reported in China, and serotypes 1, 2, and 10 have been responsible for most of the major outbreaks [8].

Up to date, several virulence-associated genes, including VapD, CAMP cohemolysis, outer membrane protein and TonB-dependent receptor tbdr1 have been identified in $R$. anatipestifer strains $[3,9,10]$. Recently, 49 novel virulence genes were identified from a transposon mutant library [11]. Lipopolysaccharide (LPS), the main component of the outer membrane of Gram-negative bacteria, is a potent stimulant of innate immune response [12]. The LPS is composed of three distinct components: lipid A, O-antigen and core oligosaccharide. The O-antigen consists of oligosaccharide repeating units (O units), which usually contain two to eight residues from a broad range of sugars, 
both common and rare, and their derivatives. The diversity of the O-antigen repeats is displayed in the types of sugar conformation, their arrangement, and the linkages within and between $\mathrm{O}$-units $[13,14]$. Consequently, the O-antigen repeats are the most variable constituent of the LPS molecule, imparting the antigenic specificity.

The O-antigen repeats are synthesized in the cytoplasm and then transported to the periplasmic face of the inner membrane. In Escherichia coli and Salmonella enteric, genes required for the biosynthesis of $\mathrm{O}$-antigen repeats are located between galF and gnd genes [15]. In R. anatipestifer, however, three genes of the AS87_04050, M949_1556 and M949_1603 were identified to be involved in O-antigen biosynthesis [16-18]. In this study, we obtained one mutant strain RA1067 that lost the reactivity with anti-LPS MAb by screening a random Tn4351 transposon insertion library using a monoclonal antibody against $R$. anatipestifer serotype 1 LPS (anti-LPS MAb). Sequence analysis showed that the M949_RS01915 gene was inactivated in the RA1067. Furthermore, the bacterial antigenicity, pathogenicity and gene expression of the RA1067 were characterized.

\section{Materials and methods}

\section{Ethics}

One-day old Cherry Valley ducks were obtained from Zhuang Hang Duck Farm (Shanghai, China) and reared under controlled temperature $\left(28-30{ }^{\circ} \mathrm{C}\right)$. The ducks were accommodated in cages with free access to food and water under the conditions of biological safety. Animal experiments were carried out in agreement with the Institutional Animal Care and Use Committee (IACUC) guidelines set by Shanghai Veterinary Research Institute, the Chinese Academy of Agricultural Sciences (CAAS). This animal study protocol (Shvri-po-0176) was approved by the IACUC of Shanghai Veterinary Research Institute, CAAS, China.

\section{Bacterial strains, plasmids and culture conditions}

The bacterial strains and plasmids used in this study are listed in Table 1. R. anatipestifer $\mathrm{CH} 3$ is the serotype 1, wild-type (WT) strain used in this study, and the mutant strain RA1067 was obtained from this strain by Tn4351 insertion. $R$. anatipestifer strains were grown on Tryptic Soy Agar (TSA, Difco, NJ, USA) or in Tryptic Soy Broth (TSB, Difco) at $37{ }^{\circ} \mathrm{C}$ with $5 \% \mathrm{CO}_{2}$. Escherichia coli strains were grown at $37{ }^{\circ} \mathrm{C}$ on Luria-Bertani (LB) plates or in LB broth. Antibiotics were used at the given concentrations when required: ampicillin $(100 \mu \mathrm{g} / \mathrm{mL})$, chloramphenicol $(30 \mu \mathrm{g} / \mathrm{mL})$, erythromycin $(0.5 \mu \mathrm{g} / \mathrm{mL})$, kanamycin $(50 \mu \mathrm{g} / \mathrm{mL})$, streptomycin $(50 \mu \mathrm{g} / \mathrm{mL})$ and cefoxitin $(5 \mu \mathrm{g} / \mathrm{mL})$.

\section{Indirect ELISA}

The anti-LPS MAb 8A9 was used to screen the Tn4351 insertion mutants for loss of reactivity by an indirect ELISA [18]. Briefly, 96-well ELISA plates were coated with the mutant strain suspended in carbonate buffered saline (CBS, pH 9.6) at $10^{9} \mathrm{CFU} /$ well in $50 \mu \mathrm{L}$, and then heat-dried overnight in a drying oven at $55{ }^{\circ} \mathrm{C}$. After being washed three times with phosphate-buffered saline (PBS) containing $0.05 \%$ Tween 20 (PBST), the plates were blocked for $2 \mathrm{~h}$ at $37^{\circ} \mathrm{C}$ in PBS containing 5\% skim milk, washed with PBST, and then incubated for $2 \mathrm{~h}$ with the anti-LPS MAb, followed by incubation for $1.5 \mathrm{~h}$ with a horseradish peroxidase (HRP)-conjugated anti-mouse IgG polyclonal antibody (Tiangen, Beijing, China). The reaction was visualized by addition of $3,3^{\prime}, 5,5^{\prime}$-tetramethyl benzidine (TMB) (Tiangen) and stopped using $2 \mathrm{M} \cdot \mathrm{H}_{2} \mathrm{SO}_{4}$ solution. The resulting $\mathrm{OD}_{450}$ values were obtained using a plate reader (Synergy 2; BioTeck). The mutant with the $\mathrm{OD}_{450}$ value of $<2.1$ times of negative wells was selected for further analysis. All the mutants were screened in triplicate. The WT strain $\mathrm{CH} 3$ was used as a positive coating control.

\section{Identification of a mutant strain}

Polymerase chain reaction (PCR) was performed to identify the WT strain CH3 and mutant strain RA1067 using primers 16S rRNA F/16S rRNA R, Erm-F/Erm-R and RA1067-F/RA1067-R (Table 1). Southern blot analysis was used to identify transposon Tn4351 insertion in the mutant strain genome [19]. Briefly, genomic DNA of the mutant strain was extracted using TIANamp Bacteria DNA kit (Tiangen), digested by XbaI, separated by gel electrophoresis, and transferred to a nylon membrane. After being washed with saline sodium citrate, the membrane was immobilized for $2 \mathrm{~h}$ at $80{ }^{\circ} \mathrm{C}$. A probe was prepared using a DIG DNA labeling and detection kit (Roche, Indianapolis, IN, USA). Southern blot hybridization was performed by standard method in accordance with the manufacturer's instructions. The plasmid pEP4351 and genomic DNA of the WT strain CH3 were also subjected to hybridization analysis, which were used as the positive and negative controls respectively.

Inverse PCR was used to determine the transposon insertion site in the mutant strain [20]. Briefly, genomic DNA of the mutant strain was digested with HindIII and ligated to form a closed circle. The DNA adjacent to the insertion site was amplified using Tn4351-specific primers TN-1 and IS4351-F. DNA sequencing data were compared to a database using BLAST from the National Center for Biotechnology Information (NCBI) website [21]. 
Table 1 Strains, plasmids and primers used in this study

\begin{tabular}{|c|c|c|}
\hline Strains, plasmids or primers & Characteristics & Source or references \\
\hline \multicolumn{3}{|l|}{ Strains } \\
\hline $\mathrm{CH} 3$ & Riemerella anatipestifer serotype 1 strain & {$[8]$} \\
\hline RA1067 & Tn4351 insertion at M949_RS01915 gene mutant of Riemerella anatipestifer CH3 & This study \\
\hline BW19851 (pEP4351) & Plasmid pEP4351 in BW19851, CmR & [8] \\
\hline WJ4 & R. anatipestifer wild-type strain, serotype 1 & {$[8]$} \\
\hline Yb2 & R. anatipestifer wild-type strain, serotype 2 & {$[8]$} \\
\hline HXb2 & R. anatipestifer wild-type strain, serotype 10 & {$[8]$} \\
\hline \multicolumn{3}{|l|}{ Primers } \\
\hline $16 \mathrm{~S}$ rRNA F & 5'-GAGCGGTAGAGTATCTTCGGATACT-3' & This study \\
\hline 165 rRNA R & 5'-AATTCCTTTGAGTTTCAACCTTGCG-3' & This study \\
\hline TN-1 & 5'-GGACCTACCTCATAG-3' & This study \\
\hline IS4351 & $5^{\prime}$-TCAGAGTGAGAGAAAGGG-3' & This study \\
\hline Tn4351-F & 5'-TGGCACCTTTGTGGTTCTTAC-3' & This study \\
\hline Tn4351-R & 5'-GAGAGACAATGTCCCCCTTTC-3' & This study \\
\hline Erm-F & 5'-GCCCGAAATGTTCAAGTTGT-3' & This study \\
\hline Erm-R & 5'-CTTGACAACCACCCGACTTT-3' & This study \\
\hline M949_RS01915F & 5'-AGTCTGCGTTGATCACCTTT-3' & This study \\
\hline M949_RS01915R & 5'-AGCAAATACCAACAGAAGGGA-3' & This study \\
\hline M949_RS01920F & 5'-AACCTCACTATCGGACCAGG-3' & This study \\
\hline M949_RS01920R & 5'-GCCCGTTCCCAATTATTTGC-3' & This study \\
\hline M949_RS01910F & 5'-GGAACTGGGATAGACGACCA-3' & This study \\
\hline M949_RS01910R & 5'-AGAGCAGAGCGTTTACCCTT-3' & This study \\
\hline RA Idh- F & 5'-AGAGGAGCTTATCGGCATCA-3' & This study \\
\hline RA Idh - R & 5'-CTAGGGCTTCTGCCAATCTG-3' & This study \\
\hline RA1067-F & 5'-ATGAATTATTTTAAACTGCT-3' & This study \\
\hline RA1067-R & 5'-TTAGTCTAATTTCTGTATAT-3' & This study \\
\hline
\end{tabular}

\section{LPS extraction, silver staining and Western blot}

LPS was extracted from the WT strain $\mathrm{CH} 3$ and mutant strain RA1067 according to the instructions of the manufacturer of the LPS extraction Kit (iNtRON Biotechnology, Boca Raton, FL, USA). Purified LPS was analyzed by sodium dodecyl sulfate polyacrylamide gel electrophoresis (SDS-PAGE). Gels were stained with silver to visualize the presence of LPS [22], and stained with coomassie blue to exclude the contamination of protein.

For Western blot analysis, the purified LPS were separated by SDS-PAGE and then transferred onto nitrocellulose membranes (Millipore, Billerica, MA, USA). The membranes were blocked overnight at $4{ }^{\circ} \mathrm{C}$ in PBS containing 5\% skim milk, washed with PBST and then incubated for $2 \mathrm{~h}$ with anti-LPS MAb, followed by incubation for $1 \mathrm{~h}$ with an IRDYE680CW-conjugated donkey antimouse IgG polyclonal antibody (LI-COR Biosciences, Lincoln, NE, USA). The blots were visualized with an Odyssey two-color infrared imaging system (LI-COR Biosciences).

\section{Adhesion and invasion assays}

Adhesion and invasion assays were performed with Vero cells (ATCC CCL-81) as described [3]. Briefly, Vero cells $\left(10^{5} /\right.$ well $)$ were seeded into 24 -well tissue culture trays in Dulbecco's modified Eagle medium (DMEM), containing $10 \%$ fetal bovine serum (FBS, Biowest, France). Vero cells were grown for $24 \mathrm{~h}$ at $37^{\circ} \mathrm{C}$ in a humidified incubator with $5 \% \mathrm{CO}_{2}$ atmosphere, rinsed three times with sterile PBS and infected with approximately $10^{7} \mathrm{CFU}$ of each strain, respectively. The infected cells were then incubated at $37^{\circ} \mathrm{C}$ with $5 \% \mathrm{CO}_{2}$ for $1.5 \mathrm{~h}$, rinsed three times with sterile $\mathrm{PBS}$ and lysed with $0.1 \%$ trypsin $(100 \mu \mathrm{L} /$ well $)$. The number of celladherent bacteria was determined after tenfold dilution and spreading onto TSA plates. For the invasion assay, extracellular bacteria were killed with $100 \mu \mathrm{g} / \mathrm{mL}$ gentamicin in DMEM medium by additional 1-h incubation after bacterial infection. After being washed three times with sterile PBS, the infected cells were lysed and the amount of intracellular bacteria was counted. All of the above assays were tested in triplicate and replicated three times. 


\section{Bacterial growth curves and virulence determination}

The growth curves of the WT strain $\mathrm{CH} 3$ and mutant strain RA1067 were measured as described previously [8]. The WT strain $\mathrm{CH} 3$ and mutant strain RA1067 were grown in TSB respectively at $37{ }^{\circ} \mathrm{C}$ for $8 \mathrm{~h}$ with shaking. The bacterial cultures were then inoculated into fresh TSB medium at a ratio of $1: 100(\mathrm{v} / \mathrm{v})$ and incubated at $37{ }^{\circ} \mathrm{C}$, with shaking at $200 \mathrm{rpm}$. Bacterial growth was measured by counting the number of bacterial CFU at $2 \mathrm{~h}$ intervals for $16 \mathrm{~h}$.

To determine whether the M949_RS01915 gene plays a role on virulence of $R$. anatipestifer, the bacterial median lethal doses $\left(\mathrm{LD}_{50}\right)$ of the WT strain $\mathrm{CH} 3$ and mutant strain RA1067 were determined using 18-day-old Cherry Valley ducks as described [3]. The ducks were evenly divided into five groups (8 ducks/group), and injected intramuscularly with the bacterial strain at a dose of $10^{6}, 10^{7}, 10^{8}, 10^{9}$, or $10^{10} \mathrm{CFU}$, respectively. Moribund ducks were euthanized humanely with an intravenous injection of sodium pentobarbital at a dose of $120 \mathrm{mg} /$ $\mathrm{kg}$ and counted as dead. Dead ducks were subjected to $R$. anatipestifer identification. Ducks were monitored daily for clinical symptoms and death rate for a period of 7 days post-infection. $\mathrm{LD}_{50}$ value was calculated by the improved Karber's method [23].

Seventeen-day-old Cherry Valley ducks were injected intramuscularly with $10^{8} \mathrm{CFU}$ of the WT strain $\mathrm{CH} 3$ and mutant strain RA1067 to evaluate the bacterial survival in vivo. Blood samples were collected at 6, 12, 24 and $48 \mathrm{~h}$ after infection (six ducks per group at each time point), were diluted tenfold and plated on TSA plates for bacterial counting.

\section{Serum sensitivity assays}

Bacterial susceptibility to normal duck sera was conducted as described [24], with modifications. Briefly, normal duck sera were collected from the 17-old-day healthy Cherry Valley ducks, pooled and filter-sterilized (0.22 um). Pooled duck sera were diluted to 12.5, 25, 50\% $(\mathrm{v} / \mathrm{v})$ in $\mathrm{pH}$ 7.2 PBS. Each $10 \mu \mathrm{L}$ of bacterial suspension containing $10^{8} \mathrm{CFU}$ was added into $190 \mu \mathrm{L}$ serial diluted duck sera, pooled duck sera without dilution, the heatinactivated duck sera $\left(56{ }^{\circ} \mathrm{C}, 30 \mathrm{~min}\right)$ and PBS, respectively. The reaction mixtures were incubated at $37{ }^{\circ} \mathrm{C}$ with $5 \% \mathrm{CO}_{2}$ for $30 \mathrm{~min}$, and then tenfold serial diluted and plated onto TSA plates. The plates were incubated at $37{ }^{\circ} \mathrm{C}$ with $5 \% \mathrm{CO}_{2}$ for $28 \mathrm{~h}$ to count bacterial colonies.

\section{Cross-protection assessment}

The inactivated vaccine was developed using the mutant strain RA1067 to evaluate the cross-protection. The inactivated $\mathrm{CH} 3$ vaccine was used as the WT strain control and developed as described [17]. A total of 72 ducks (7-day old) were divided into three groups of 24, received two immunizations at day 7 and 21 respectively with inactivated RA1067 vaccine (group 1, $5 \times 10^{8} \mathrm{CFU}$ bacterial cells in $0.3 \mathrm{~mL}$ vaccine), inactivated $\mathrm{CH} 3$ vaccine (group 2, $5 \times 10^{8} \mathrm{CFU}$ bacterial cells in $0.3 \mathrm{~mL}$ vaccine), as described [25]. The ducks in group 3 received two subcutaneous injections of saline in adjuvant as controls. At 2 weeks post-immunization, each eight ducks from each group were challenged with $R$. anatipestifer strain WJ4 (serotype 1), Yb2 (serotype 2) or HXb2 (serotype 10) by subcutaneous injection at a dose of $10 \mathrm{LD}_{50}$ in $0.5 \mathrm{~mL}$ saline, respectively. Ducks were monitored and recorded daily for clinical symptoms and death until 7 days post-infection.

\section{Illumina sequencing for RNA-Seq and differential expression analysis}

Total RNA quantification and quality were assessed by spectrophotometer, ribosome RNA were removed using Ribo-Zero ${ }^{\mathrm{TM}}$ Magnetic Gold Kit (epicenter, USA), then the protocol of TruSeq RNA Sample Prep Kit v2 (Illumina) to construct the libraries was followed. The complete libraries were sequenced for 100 cycles on Illumina HiSeq 2000 as described [26]. Image analysis and base calling were performed using Solexa pipeline Version 1.8 (Off-Line Base Caller software, Version 1.8) [27]. Cleaned reads were aligned to $R$. anatipestifer $\mathrm{CH} 3$ genome using RNA Sequel software [28]. Transcript levels were calculated as RPKM (Reads per kilobase cDNA per million fragments mapped). Differential expressed genes were analyzed using Cufflinks software (version 2.1.1) with fold change (cutoff $=2.0$ ) [29], and considered statistically significant if the fold change was $>2.0$ and the FDR (False Discovery Rate) was $<0.001$.

\section{Real-time quantitative PCR analysis}

Real-time qPCR was performed to confirm transcriptional levels of differently expressed genes obtained in the RNA-Seq analysis. Gene-specific primers were designed using primer3 online software Version.0.4.0 [30] and described in Table 1. The expression of the L-lactate dehydrogenase encoding gene (ldh) was measured using primers RA ldh-F/RA ldh-R (Table 1), and used as an internal control [31]. Total RNA was isolated from the WT strain CH3 and mutant strain RA1067 using Trizol reagent (Invitrogen, Carlsbad, CA, USA), according to the manufacturer's instructions. All RNA samples were treated with TURBO DNA-free kit (Ambion, Grand Island, NY, USA) to remove DNA contamination. cDNA was synthesized using PrimeScript RT Master Mix (Takara). Real-time qPCR was carried out in Go Taq qPCR Master Mix (Promega, Fitchburg, WI, USA) using the following parameters: $95{ }^{\circ} \mathrm{C}$ for $2 \mathrm{~min}, 40$ cycles of 
$95{ }^{\circ} \mathrm{C}$ for $15 \mathrm{~s}, 55^{\circ} \mathrm{C}$ for $15 \mathrm{~s}$ and $68^{\circ} \mathrm{C}$ for $20 \mathrm{~s}$, followed by one cycle of $95{ }^{\circ} \mathrm{C}$ for $15 \mathrm{~s}, 60{ }^{\circ} \mathrm{C}$ for $15 \mathrm{~s}$ and $95{ }^{\circ} \mathrm{C}$ for $15 \mathrm{~s}$. Reactions were performed in triplicate and run on the Mastercycler ep realplex4 apparatus (Eppendorf, Germany). Quantification of transcriptional level was calculated according to the $2^{-\Delta \Delta C t}$ method.

\section{Statistical methods}

Statistical analyses were performed using the GraphPad Prism, version 5.0 for Windows (GraphPad Software Inc., La Jolla, CA, USA). Adhesion and invasion assays, bacterial growth curves, bacterial loads in the blood of ducks, serum sensitivity assays, and RT-PCR were two tailed, and a $p$ value of $<0.05$ was considered significant. Multigroup comparisons were carried out using ANOVA.

\section{Results}

\section{Identification of the mutant strain RA1067}

The mutant RA1067 that lacked reactivity with anti-LPS MAb was obtained by screening the transposon library using an indirect ELISA, and identified by PCR amplification using primers 16S rRNA F/16S rRNA R, Erm-F/ Erm-R and RA1067-F/RA1067-R. As shown in Figure 1A, a 744-bp fragment of $16 \mathrm{~S}$ rRNA was amplified from the WT strain $\mathrm{CH} 3$ (lane 1) and the mutant strain RA1067 (lane 2); a 714-bp fragment of M949_RS01915 gene was amplified from the WT strain CH3 (lane 7), while no 714-bp fragments were amplified from the mutant strain RA1067 due to the transposon insertion (lane 8); a 644-bp fragment of the erm gene (Tn4351 transposon contained the erm gene) was amplified from the mutant strain RA1067 (lane 5), but not from the WT strain CH3 (lane 4). Southern blot indicated that the mutant strain RA1067 contained a single Tn4351 insertion in the chromosomal DNA (Figure 1B, lane 2). Sequencing analysis showed that the transposon was inserted at nucleotide position 116 bp of M949_RS01915 gene, which consists of 714 nucleotides and encodes 237 amino acids (Figure 1C). Real-time qPCR analysis further confirmed M949_RS01915 transcription was abolished in the mutant strain RA1067. Further investigation showed that the gene deletion had no effect on the transcription of chromosomally upstream M949_RS01920 gene or downstream M949_RS01910 gene (Figure 1D). BLAST analysis showed that the M949_RS01915 gene was highly conserved in $R$. anatipestifer, which exhibits over $91 \%$ identity compared to other $R$. anatipestifer strains.

\section{Analysis of the bacterial LPS by silver staining and western blot}

LPS was purified from the WT strain $\mathrm{CH} 3$ and the mutant strain RA1067, and followed by silver staining and Western blot analyses. As shown in Figure 2A, LPS purified from the WT strain $\mathrm{CH} 3$ displayed a ladderlike pattern at $70 \mathrm{kDa}$, while it was absent in the mutant strain RA1067 LPS in the silver staining. No detectable protein bands were found in the gels by Coomassie blue staining (data not shown). Western blot analysis showed that a ladder-like pattern of $\mathrm{O}$-antigen repeats to antiLPS MAb was defected in the mutant strain RA1067 (Figure 2B). These results are beginning to suggest that the $R$. anatipestifer M949_RS01915 gene is involved in LPS O-antigen biosynthesis.

\section{Adhesion and invasion assays}

To determine whether disruption of M949_RS01915 gene affects the bacterial adherence and invasion capacity, we compared bacterial adhesion and invasion abilities of the CH3 and RA1067 strains on Vero cells. The host cellassociated bacteria of the WT strain $\mathrm{CH} 3$ and mutant strain RA1067 were counted as $2.2 \times 10^{4} \mathrm{CFU} /$ well and $3.46 \times 10^{5} \mathrm{CFU} /$ well respectively when infected at 50 multiplicity of infection (MOI), and $3.49 \times 10^{4} \mathrm{CFU} /$ well and $8.05 \times 10^{5} \mathrm{CFU} /$ well respectively when infected at 100 MOI. The mutant strain RA1067 presented a 15.73or 23.07-fold increased adherence ability in comparison with those of the WT strain CH3 $(p<0.001)$ (Figure 3A). After further 1 -h incubation with $100 \mu \mathrm{g} / \mathrm{mL}$ gentamycin, invaded bacterial CFU of the mutant strain RA1067 were $1.41 \times 10^{5} \mathrm{CFU} /$ well at MOI of 50, and $2.87 \times 10^{5} \mathrm{CFU} /$ well at an MOI of 100, which were 19.58- and 26.57-fold higher than those of the WT strain CH3 $\left(7.2 \times 10^{3} \mathrm{CFU} /\right.$ well at an MOI of $50 ; 1.08 \times 10^{4} \mathrm{CFU} /$ well at an MOI of 100) $(p<0.001)$ (Figure $3 \mathrm{~B})$. The results showed that adhesion and invasion capacities of the mutant strain RA1067 were significantly increased in comparison with those of the WT strain $\mathrm{CH} 3$.

\section{Determination of bacterial growth curves and virulence}

The growth of the mutant strain RA1067 was significantly decreased at the late stage of the growth when compared to those of the WT strain $\mathrm{CH} 3$. The mutant strain RA1067 reached the plateau of $1.32 \times 10^{9} \mathrm{CFU} /$ $\mathrm{mL}$ at 8 -h growing in TSB, while the WT strain $\mathrm{CH} 3$ reached the plateau of $3.68 \times 10^{9} \mathrm{CFU} / \mathrm{mL}$ at 12 -h growing in TSB. The bacterial numbers of the mutant strain RA1067 at the plateau was about 2.79-fold decreased in comparison with that of the WT strain CH3 (Figure 4A). Bacterial virulence was evaluated based on bacterial $\mathrm{LD}_{50}$ determination using 17-day-old Cherry Valley ducks. The $\mathrm{LD}_{50}$ for the mutant strain RA1067 was $2.74 \times 10^{10}$ CFU, which was more than 365 times attenuated virulence than that of the WT strain $\mathrm{CH} 3\left(7.50 \times 10^{7} \mathrm{CFU}\right)$. In addition, the bacterial loading experiment further confirmed the attenuated virulence of the mutant strain RA1067. As shown in Figure 4B, the bacterial loads in the 


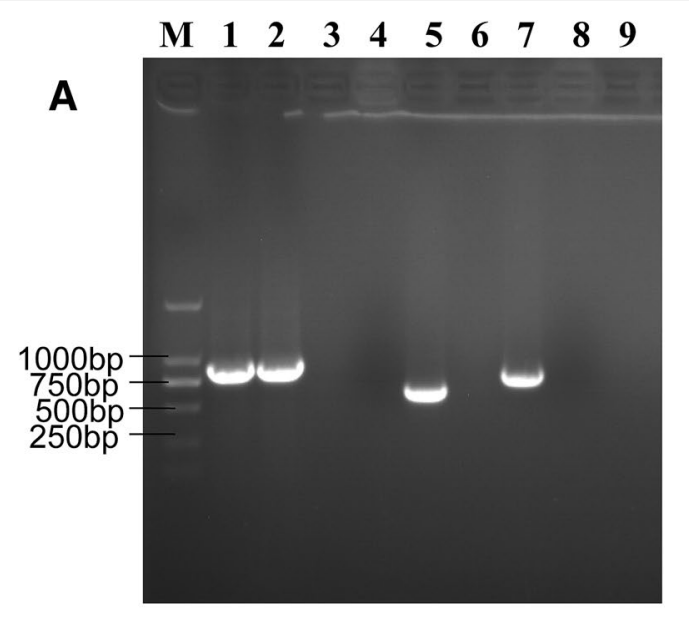

C

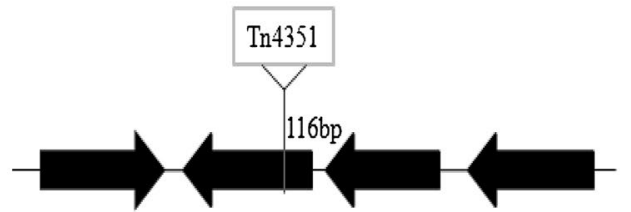

M949_RS01910 M949_RS01915 M949_RS01920 M949_RS01925

(714bp)
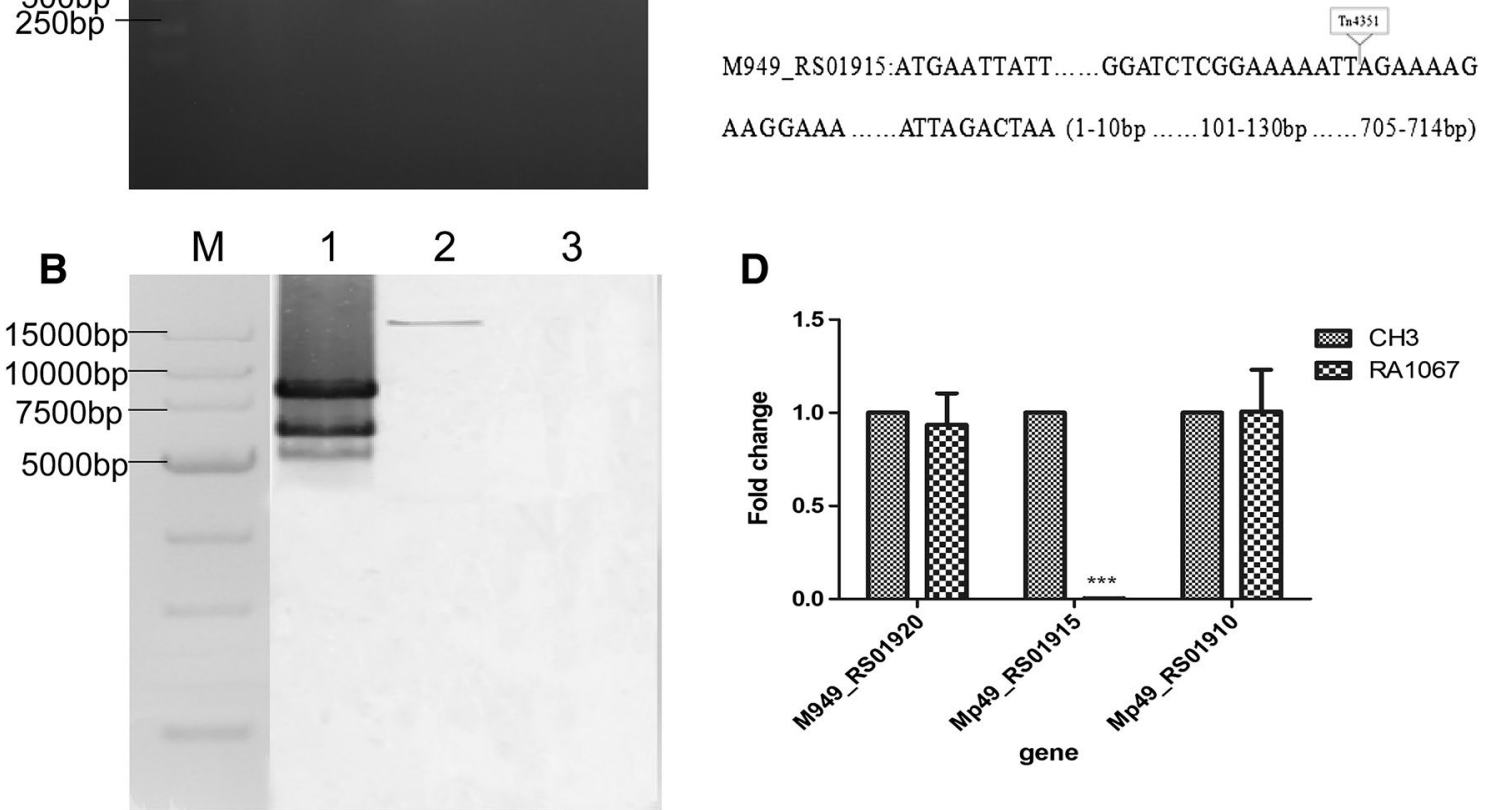

D

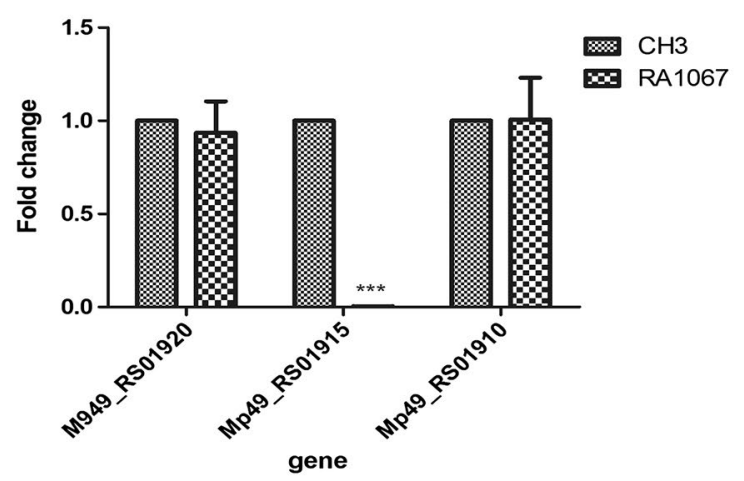

Figure 1 Identification of the mutant strain RA1067. A PCR amplification. M: Takara DL2000 marker; lanes 1-2: R. anatipestifer 16S rRNA was amplified from the WT strain CH3 (lane 1), the mutant strain RA1067 (lane 2), showing a 744-bp fragment of 16S rRNA; lanes 4-5: 644 bp fragment of the erm gene was amplified from the mutant strain RA1067 (lane 5), but not from the WT strain CH3 (lane 4); lanes 7-8: 714-bp fragment of M949_ RS01915 gene was amplified from the WT strain CH3 (lane 7), but not from the mutant strain RA1067 (lane 8); lanes 3, 6 and 9 distilled water, as negative controls. B Southern blot analysis of the transposon Tn4351 insertion. Lane M Takara DL15000 marker; Lane 1, $10 \mu \mathrm{g}$ of pEP4351 digested with Xbal (positive control). Lane 2, $10 \mu \mathrm{g}$ of chromosomal DNA from mutant strain RA1067 digested with Xbal; Lane 3, $10 \mu \mathrm{g}$ of chromosomal DNA from the WT strain $\mathrm{CH} 3$ digested with Xbal (negative control); each digested sample was resolved on a $0.7 \%$ agarose gel, and Southern blot was performed using a TnDIG labeled probe. C Schematic chart of Tn4351 insertion in the RA1067 chromosome. D Real time qPCR analysis. The changes of mRNA were expressed as fold expression and calculated using the comparative $C_{T}\left(2^{-\Delta \Delta C T}\right)$ method. Data were normalized to the housekeeping gene $/ d h$ and expressed as fold changes. Error bars represent standard deviations from three replicates $(* * *, p<0.001)$.

blood of ducks infected with the mutant strain RA1067 were decreased significantly in comparison with those of the ducks infected with the WT strain $\mathrm{CH} 3$ at $48 \mathrm{~h}$ post infection (hpi) $(p<0.001)$.

\section{The mutant strain RA1067 displayed a higher sensitivity to normal duck sera}

To determine whether the M949_RS01915 gene is involved in serum resistance of the WT strain $\mathrm{CH} 3$, we compared the WT strain $\mathrm{CH} 3$ and mutant strain RA1067 for their abilities to resist the complement-mediated killing. The results showed that $25 \%$ diluted serum was effective in killing the mutant strain RA1067, but not the WT strain $\mathrm{CH} 3$, indicating that the mutant strain RA1067 was more sensitive to normal duck sera than that of the WT strain $\mathrm{CH} 3$ (Figure 5).

\section{Cross-protection experiment}

To investigate the cross-protection against $R$. anatipestifer serotypes 1,2 and 10 strains, the RA 1067 vaccinated ducks were challenged with virulent $R$. anatipestifer strains WJ4 (serotype 1), Yb2 (serotype 2) and HXb2 (serotype 10 ) at $10 \mathrm{LD}_{50}$ respectively. When challenged with strains $\mathrm{WJ} 4, \mathrm{Yb} 2$, or $\mathrm{HXb} 2$ at day 14 post 

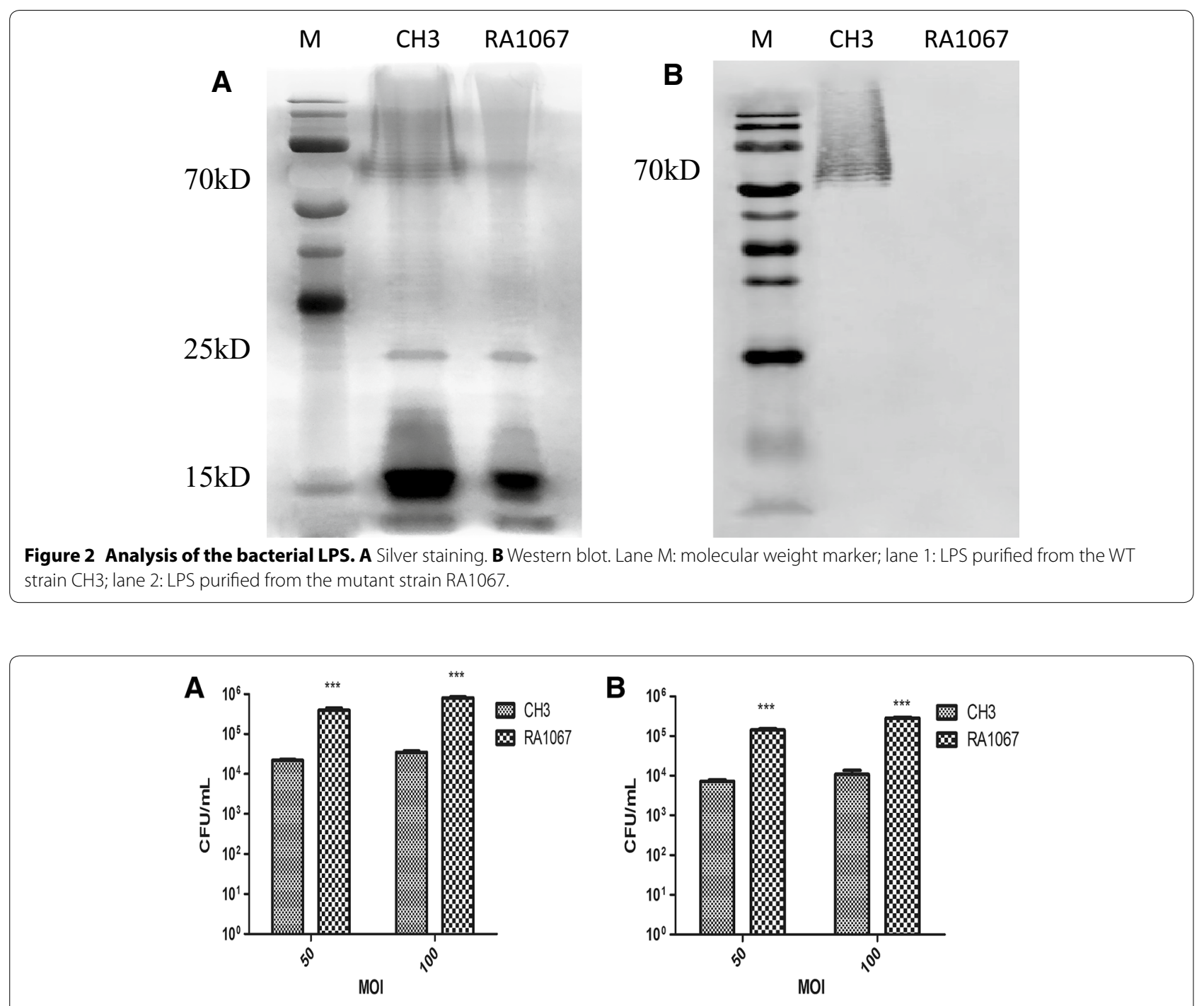

Figure 3 Bacterial adherence and invasion assays. Strains $\mathrm{CH} 3$ and RA1067 were tested on Vero cells. A Adherence assay; B invasion assay. The data represent the number of bacteria bound to or invaded into Vero cells in each well of a 24-well plate. The error bars represent mean \pm standard deviations from three independent experiments $\left({ }^{* * *}, p<0.001\right)$.

vaccination, the ducks in group 1 were $7 / 8,6 / 8$, or $7 / 8$ protected respectively; the ducks in group 2 were $7 / 8$, $2 / 8$, or $5 / 8$ protected respectively. The protection rate of the WT strain $\mathrm{CH} 3$ vaccine against WJ4 challenge was the same as that of the mutant strain, however, the protection against $\mathrm{Yb} 2$ and $\mathrm{HXb} 2$ challenges was 50 and $25 \%$ lower than those of the mutant strain RA 1067 vaccine respectively. The ducks in group 3 (control group) were all dead within 7 days post challenge (Table 2). These results indicate that RA1067 provided cross-protection against challenge with strains from $R$. anatipestifer serotypes 1, 2 and 10, further confirmed its altered antigenicity.

\section{Determination of the differentially expressed genes}

Strand-specific Illumina RNA-Seq analysis was used to investigate the differentially expressed genes between the WT strain $\mathrm{CH} 3$ and mutant strain RA1067. In total, 9 genes were up-regulated and 10 genes were downregulated in the mutant strain RA1067 in comparison to the WT strain $\mathrm{CH} 3$ (Table 3), which were differentially expressed by $>$ fivefold based on RNA-Seq analysis. Real-time qPCR further confirmed M949_RS01940, M949_RS09405 genes were up-regulated over twofold at transcriptional levels. The proteins encoded by M949_RS01940 and M949_RS09405 genes were annotated as hypothetical protein and transcriptional 

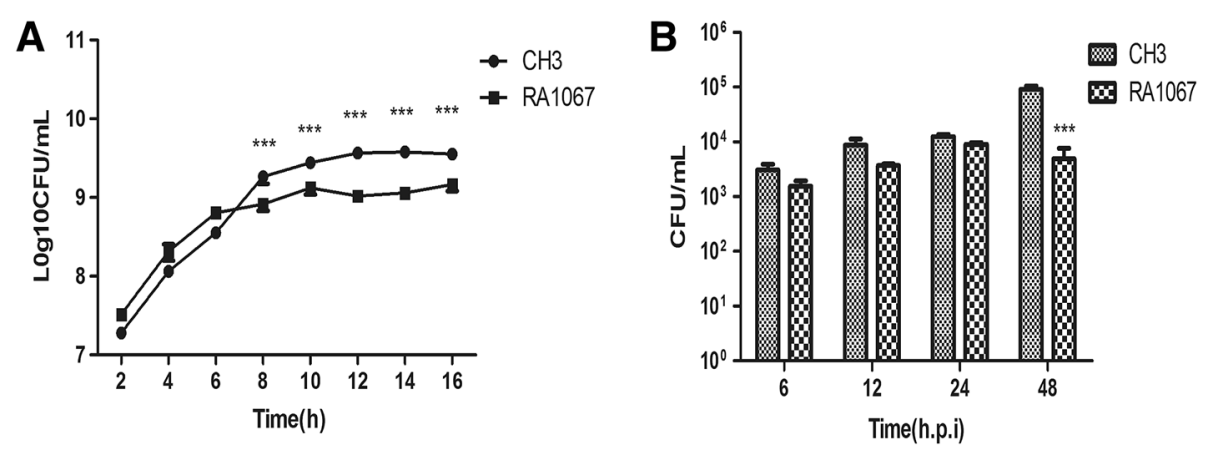

Figure 4 Determination of the bacterial growth curves and virulence. A Strains $\mathrm{CH} 3$ and RA 1067 were grown in $\mathrm{TSB}$ at $37^{\circ} \mathrm{C}$ with shaking respectively, and the bacteria numbers of CFU were measured at $2 \mathrm{~h}$ intervals. This experiment was repeated three times, and the data were presented as the means. Error bars represent standard deviations. Asterisks indicate statistically significant differences between groups. B Bacterial loads in blood of infected ducks with $\mathrm{CH} 3$ or RA1067 at 6, 12, 24 and 48 h post infection (hpi). After 72 hpi more than half of the WT CH3 infected ducks were dead, no data of bacterial loads in blood was collected. The error bars represent means \pm standard deviations from six ducks. Asterisks indicate statistically significant differences between groups $(* * *, p<0.001)$.

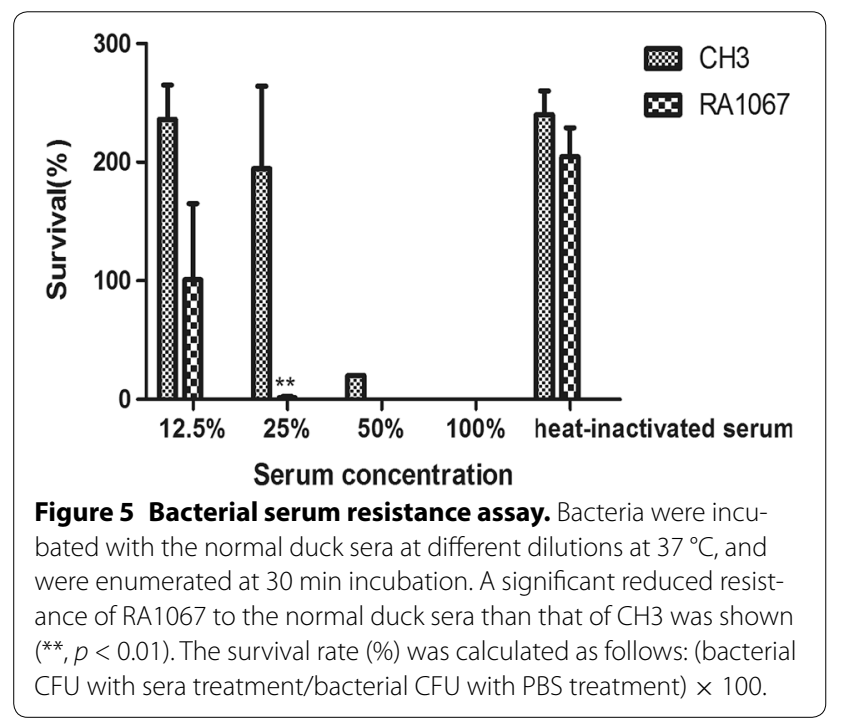

regulator respectively. Three genes of M949_RS07580, M949_RS10455 and M949_RS00790 were further verified to be down-regulated over twofold at transcriptional levels. The coding products were annotated as polysaccharide biosynthesis protein CapD, DNA-binding protein and galactose-binding protein respectively. The polysaccharide biosynthesis protein CapD is responsible for bacterial capsule polysaccharide biosynthesis. The results indicate that the M949_RS01915 gene may regulate the genes mainly responsible for bacterial polysaccharide biosynthesis.

\section{Discussion}

DNA transposition was used as a powerful approach for the generation of appropriate knockout mutations for functional gene analysis. In a previous study, random transposon mutagenesis was used to successfully identify

Table 2 Cross-protection experiment

\begin{tabular}{|c|c|c|c|}
\hline Groups & Challenge strains & Total no. of ducks & Protection rate (protected no./total no.) \\
\hline \multirow{3}{*}{$\begin{array}{l}1 \\
\text { (RA1067 vaccine) }\end{array}$} & WJ4 & 8 & $7 / 8$ \\
\hline & Yb2 & 8 & $6 / 8$ \\
\hline & $\mathrm{HXb} 2$ & 8 & $7 / 8$ \\
\hline \multirow{3}{*}{$\begin{array}{l}2 \\
\text { (CH3 vaccine) }\end{array}$} & WJ4 & 8 & $7 / 8$ \\
\hline & $\mathrm{Yb} 2$ & 8 & $2 / 8$ \\
\hline & $\mathrm{HXb2}$ & 8 & $5 / 8$ \\
\hline \multirow{3}{*}{$\begin{array}{l}3 \\
\text { (Saline in adjuvant) }\end{array}$} & WJ4 & 8 & 0 \\
\hline & Yb2 & 8 & 0 \\
\hline & $\mathrm{HXb} 2$ & 8 & 0 \\
\hline
\end{tabular}

a Challenge dose was $10 \mathrm{LD}_{50}$ for each strain. 
Table 3 Real-time qPCR verification of differentially expressed genes in mutant strain RA1067

\begin{tabular}{|c|c|c|}
\hline Gene locus $^{a}$ & Description of genes & $2^{-\Delta \Delta C t}$ \\
\hline M949_RS01940 & Hypothetical protein & 3.71 \\
\hline M949_RS09405 & Transcriptional regulator & 3.09 \\
\hline M949_RS10515 & Hypothetical protein & 1.92 \\
\hline M949_RS00810 & Hypothetical protein & 1.88 \\
\hline M949_RS04680 & TonB-dependent receptor & 1.46 \\
\hline M949_RS03025 & TonB-denpendent receptor & 1.03 \\
\hline M949_RS10460 & Hypothetical protein & 0.99 \\
\hline M949_RS09795 & Hypothetical protein & 0.88 \\
\hline M949_RS00955 & Hypothetical protein & 0.84 \\
\hline M949_RS00980 & Hypothetical protein & 0.72 \\
\hline M949_RS05615 & DNA-binding protein & 0.71 \\
\hline M949_RS05620 & Hypothetical protein & 0.68 \\
\hline M949_RS00870 & Head morphogenesis protein & 0.67 \\
\hline M949_RS07550 & thij/pfpi domain-containing protein & 0.61 \\
\hline M949_RS10465 & Hypothetical protein & 0.49 \\
\hline M949_RS10510 & S41 family peptidase & 0.44 \\
\hline M949_RS10455 & DNA-binding protein & 0.31 \\
\hline M949_RS00790 & Galactose-binding protein & 0.23 \\
\hline M949_RS07580 & Polysaccharide biosynthesis protein CapD & 0.02 \\
\hline
\end{tabular}

a Based on R. anatipestifer $\mathrm{CH} 3$ genome (accession number: CP006649).

33 genes involved in biofilm biosynthesis [32]. In the present report, the mutant strain RA1067 was identified by screening the library of random $\mathrm{Tn} 4351$ transposon mutants using anti-LPS MAb. The mutant lacks reactivity with anti-LPS MAb in an indirect ELISA. Further investigations revealed that the mutant showed different growth characteristics in TSB, increased capacity of adhesion and invasion, increased sensitivity to normal duck serum, and significantly attenuated virulence in ducks. Western blot indicated a distinct loss of ladder-like pattern in RA1067 mutant LPS, compared to those in CH3 LPS. BLAST searches showed that the M949_RS01915 gene is highly conserved among $R$. anatipestifer strains.

LPS isolated from the WT strain $\mathrm{CH} 3$ and mutant strain RA1067 were analyzed by SDS-PAGE followed by silver staining and Western blot. Silver staining showed that the RA1067 LPS lacked the ladder-like patterns as presented in the WT strain CH3 LPS. In addition, the destructed LPS lost the binding activity with anti-LPS MAb. The results above suggest that M949_RS01915 takes part in biosynthesis of integrated $\mathrm{O}$-antigen in $R$. anatipestifer. Consequently, we investigated the adherence and invasion capability of the RA1067 on Vero cells as described previously. The result manifested that the adhesion and invasion capacities of the RA1067 were sharply increased compared to those of the WT strain $\mathrm{CH} 3$. Moreover, the virulence of the RA1067 was more than 365 times attenuated than that of the WT strain $\mathrm{CH} 3$ based on the $\mathrm{LD}_{50}$ determination and the bacterial loads in blood of ducks infected with RA1067 were significantly decreased at $48 \mathrm{~h}$, compared with those of ducks infected with $\mathrm{CH} 3$. Therefore, we demonstrate that the pathogenicity of the RA1067 was decreased compared with that of the WT strain $\mathrm{CH}$. These findings were in agreement with previous studies describing that O-antigen of LPS is essential for bacterial adherence and virulence for Salmonella [33]. Bacterial adhesion and invasion abilities of the mutant strain RA1067 were significantly enhanced, compared to those of the WT strain. In addition, the LPS pattern was altered, suggesting that the RA1067 may take a different entry route to adhere and invade cells as previously reported [34]. $\mathrm{O}$-antigen also plays an essential role in protecting bacteria from serum complement-mediated killing [35, 36]. In this study, inactivation of the M949_RS01915 gene resulted in significantly increased sensitivity to normal duck serum killing, while the WT strain $\mathrm{CH} 3$ presented a dose-dependent resistance to the normal duck serum killing. This result has also been shown previously, indicating that the deficiency of LPS O-antigen chains of $R$. anatipestifer exhibit more sensitivity to normal duck serum killing [37].

As we all know, there is no effective cross-protection among different serotypes of $R$. anatipestifer. Our results show that the inactivated RA1076 vaccine protected the ducks from challenge with $R$. anatipestifer strains WJ4 (serotype 1), Yb2 (serotype 2) and HXb2 (serotype 10) at $7 / 8,6 / 8$ and $7 / 8$ respectively; 50 and $25 \%$ were improved compared with inactivated $\mathrm{CH} 3$ vaccine challenged with $R$. anatipestifer strains $\mathrm{Yb} 2$ (serotype 2) and HXb2 (serotype 10). It has been noted that $\mathrm{O}$-antigen, one of the most variable cell constituents, is a serotype feature of Gram-negative bacteria and defines their $\mathrm{O}$-serospecificity [38]. This study demonstrates that the inactivated vaccine provides effective cross-protection because of deficiency in O-antigen of the mutant strain RA1067, further confirming that the antigenicity of the RA1067 was altered.

RNA-Seq technology was applied to analyze the differential gene expressions in the mutant strain RA1067. In our RNA-Seq analysis, we found that deletion of $R$. anatipestifer CH3 M949_RSO1915 gene resulted in upregulation of 9 genes and down-regulation of 10 genes, respectively. Real time qPCR verification further confirmed that two genes were up-regulated and three genes were down-regulated by over twofold. Protein encoded by up-regulated M949_RS09405 is BlaI family transcriptional regulator, penicillinase repressor. As for BlaI family transcriptional regulator in Staphylococcus aureus, blaI is predicted to encode a repressor protein 
and regulate the production of both PBP 2a and 1-lactamase [39]. Protein encoded by up-regulated M949. RSO1940 gene belongs to the PhnB protein family. Many proteins of the PhnB protein family have been annotated as gene 3-demethylubiquinone-9 3-methyltransferase enzymes, which is necessary for the use of phosphonates (Pn) supporting bacterial growth on alkylphosphonates as a sole phosphorus source in Escherichia coli [40]. The product of down-regulated M949_RS10455 gene is DNA-binding protein. It has been reported that some members of DNA-binding proteins are involved in DNA binding, supercoiling and DNA compaction. In addition to architectural roles, some DNA-binding proteins also play regulatory roles in DNA replication and repair, and act as global transcriptional regulators in many bacteria [41]. Galactose binding protein encoded by the down-regulated M949_RS00790 gene belongs to a group of soluble proteins that can be found in the periplasmic space of Gram-negative bacteria; some members of these proteins are responsible for stimulating chemotaxis in low nutrient environments and participate in active transport of small molecules and ions from the periplasm to the cytoplasm [42]. Down-regulated gene M949_RS07580 was annotated to encode polysaccharide biosynthesis protein CapD, which is involved in "metabolic process, DNA binding, catalytic activity". Polysaccharide biosynthesis protein CapD, in Enterococcus faecium, was identified as a virulence factor and involved in bacterial growth, biosynthesis of surface polysaccharides [43]. It has also been reported that polysaccharide biosynthesis protein CapD is associated with the Gram-negative bacterial capsule, high-molecular-weight capsular polysaccharides are critical for bacterial resisting against opsonophagocytosis and complement-mediated killing [44, 45]. The CapD protein is required for biosynthesis of type 1 capsular polysaccharide in Staphylococcus spp, and Group 1 and 4 capsules are related to LPS O-antigens [45-47]. Based on these results, for the mutant strain RA1067, we suggest that disruption of the M949_RS01915 gene mainly leads to down-regulation of the M949_RS07580 gene, which results in reduced formation of type 1 capsular polysaccharide and LPS O-antigens. However, the mechanism of M949_RS01915 gene regulation of M949_RS07580 gene is currently unknown and needs further investigation.

Bacterial LPS is biosynthesized by several dozens of genes $[15,47]$. We previously reported three genes are associated with the $R$. anatipestifer LPS biosynthesis [16-18]. In this study, one more LPS biosynthesis associated gene M949_RS01915 was identified. We used the anti-LPS monoclonal antibody to screen the LPS mutant defective in the LPS structure to identify the
LPS biosynthesis associated genes, which will benefit for the future LPS structure analysis, as well as vaccine and pathogenesis studies. In conclusion, we identified a transposon mutant RA1067 with deletion of the M949 RS01915 gene. We demonstrate that the M949_RS01915 gene is involved in LPS O-antigen synthesis, bacterial virulence and gene regulation in $R$. anatipestifer. Further experiments will be needed to characterize the M949_ RSO1915 gene to clarify its functions and the exact mechanism in bacterial virulence and gene regulation.

\section{Competing interests \\ The authors declare that they have no competing interests.}

\section{Authors' contributions}

$Y D, X W$ and GY performed the experiments, analyzed the data and prepared the manuscript. SW, MT, JQ and TL, and CD contributed reagents, materials and analysis tools. SY designed the study and revised the manuscript. All authors read and approved the final manuscript.

\section{Acknowledgements}

This work was supported by the National Natural Science Foundation of China (31272591).

Received: 29 September 2016 Accepted: 5 December 2016 Published online: 06 February 2017

\section{References}

1. Segers P, Mannheim W, Vancanneyt M, De Brandt K, Hinz KH, Kersters K, Vandamme P (1993) Riemerella anatipestifer gen. nov., comb. nov., the causative agent of septicemia anserum exsudativa, and its phylogenetic affiliation within the Flavobacterium-Cytophaga rRNA homology group. Int J Syst Bacteriol 43:768-776

2. Han X, Hu Q, Ding S, Chen W, Ding C, He L, Wang X, Ding J, Yu S (2012) Identification and immunological characteristics of chaperonin GroEL in Riemerella anatipestifer. Appl Microbiol Biotechnol 93:1197-1205

3. Hu Q, Han X, Zhou X, Ding C, Zhu Y, Yu S (2011) OmpA is a virulence factor of Riemerella anatipestifer. Vet Microbiol 150:278-283

4. Sandhu TS, Leister ML (1991) Serotypes of 'Pasteurella' anatipestifer isolates from poultry in different countries. Avian Pathol 20:233-239

5. Pathanasophon P, Sawada T, Pramoolsinsap T, Tanticharoenyos T (1996) Immunogenicity of Riemerella anatipestifer broth culture bacterin and cell-free culture filtrate in ducks. Avian Pathol 25:705-719

6. Pathanasophon P, Sawada T, Tanticharoenyos T (1995) New serotypes of Riemerella anatipestifer isolated from ducks in Thailand. Avian Pathol 24:195-199

7. Pathanasophon P, Phuektes P, Tanticharoenyos T, Narongsak W, Sawada T (2002) A potential new serotype of Riemerella anatipestifer isolated from ducks in Thailand. Avian Pathol 31:267-270

8. Hu Q, Han X, Zhou X, Ding S, Ding C, Yu S (2010) Characterization of biofilm formation by Riemerella anatipestifer. Vet Microbiol 144:429-436

9. Chang CF, Hung PE, Chang YF (1998) Molecular characterization of a plasmid isolated from Riemerella anatipestifer. Avian Pathol 27:339-345

10. Lu F, Miao S, Tu J, Ni X, Xing L, Yu H, Pan L, Hu Q (2013) The role of TonBdependent receptor TbdR1 in Riemerella anatipestifer in iron acquisition and virulence. Vet Microbiol 167:713-718

11. Wang X, Ding C, Wang S, Han X, Yu S (2015) Whole-genome sequence analysis and genome-wide virulence gene identification of Riemerella anatipestifer strain Yb2. Appl Environ Microbiol 81:5093-5102

12. Kalynych S, Morona R, Cygler M (2014) Progress in understanding the assembly process of bacterial O-antigen. FEMS Microbiol Rev 38:1048-1065

13. Reeves PP, Wang L (2002) Genomic Organization of LPS-Specific Loci. In: Hacker J, Kaper JB (eds) Pathogenicity islands and the evolution of pathogenic microbes, vol I. Springer, Berlin Heidelberg 
14. Rojas-Macias MA, Ståhle J, Lütteke T, Widmalm G (2014) Development of the ECODAB into a relational database for Escherichia coli O-antigens and other bacterial polysaccharides. Glycobiology 3:341-347

15. Samuel G, Reeves P (2003) Biosynthesis of O-antigens: genes and pathways involved in nucleotide sugar precursor synthesis and O-antigen assembly. Carbohydr Res 338:2503-2519

16. Wang X, Ding C, Wang S, Han X, Hou W, Yue J, Zou J, Yu S (2014) The AS87_04050 gene is involved in bacterial lipopolysaccharide biosynthesis and pathogenicity of Riemerella anatipestifer. PLoS One 9:e109962

17. Zou J, Wang X, Tian M, Cao S, Hou W, Wang S, Han X, Ding C, Yu S (2015) The M949_1556 gene plays a role on the bacterial antigenicity and pathogenicity of Riemerella anatipestifer. Vet Microbiol 177:193-200

18. Zou J, Wang X, Ding C, Tian M, Han X, Wang S, Yu S (2015) Characterization and cross-protection evaluation of M949_1603 gene deletion Riemerella anatipestifer mutant RA-M1. Appl Microbiol Biotechnol 99:10107-10116

19. Alvarez B, Secades P, McBride MJ, Guijarro JA (2004) Development of genetic techniques for the psychrotrophic fish pathogen Flavobacterium psychrophilum. Appl Environ Microbiol 70:581-587

20. Alvarez B, Secades P, Prieto M, McBride MJ, Guijarro JA (2006) A mutation in Flavobacterium psychrophilum tlpB inhibits gliding motility and induces biofilm formation. Appl Environ Microbiol 72:4044-4053

21. Basic local alignment search tool [https://blast.ncbi.nlm.nih.gov/Blast.cgi]. Accessed 20 July 2015

22. Tsai CM, Frasch CE (1982) A sensitive silver stain for detecting lipopolysaccharides in polyacrylamide gels. Anal Biochem 119:115-119

23. Reed $L$, Muench $H$ (1938) A simple method of estimating fifty per cent endpoints. Am J Epidemiol 27:493-497

24. McQuillen DP, Gulati S, Rice PA (1994) Complement-mediated bacterial killing assays. Methods Enzymol 236:137-147

25. Liu H, Wang X, Ding C, Han X, Cheng A, Wang S, Yu S (2013) Development and evaluation of a trivalent Riemerella anatipestifer-inactivated vaccine. Clin Vaccine Immunol 20:691-697

26. Wang X, Yue J, Ding C, Wang S, Liu B, Tian M, Yu S (2016) Deletion of AS87_03730 gene changed the bacterial virulence and gene expression of Riemerella anatipestifer. Sci Rep 6:22438

27. Whiteford N, Skelly T, Curtis C, Ritchie ME, Lohr A, Zaranek AW, Abnizova I, Brown C (2009) Swift: primary data analysis for the Illumina Solexa sequencing platform. Bioinformatics 25:2194-2199

28. Wilson GW, Stein LD (2015) RNASequel: accurate and repeat tolerant realignment of RNA-seq reads. Nucleic Acids Res 43:e122

29. Trapnell C, Williams BA, Pertea G, Mortazavi A, Kwan G, van Baren MJ, Salzberg SL, Wold BJ, Pachter L (2010) Transcript assembly and quantification by RNA-Seq reveals unannotated transcripts and isoform switching during cell differentiation. Nat Biotechnol 28:511-515

30. Primer3 (v. 0.4.0) [http://bioinfo.ut.ee/primer3-0.4.0/]. Accessed 9 May 2016

31. Huggett J, Dheda K, Bustin S, Zumla A (2005) Real-time RT-PCR normalisation; strategies and considerations. Genes Immun 6:279-284

32. Hu Q, Zhu Y, Tu J, Yin Y, Wang X, Han X, Ding C, Zhang B, Yu S (2012) Identification of the genes involved in Riemerella anatipestifer biofilm formation by random transposon mutagenesis. PLoS One 7:e39805
33. Murray GL, Attridge SR, Morona R (2003) Regulation of Salmonella typhimurium lipopolysaccharide $\mathrm{O}$ antigen chain length is required for virulence; identification of FepE as a second Wzz. Mol Microbiol 47:1395-1406

34. Zhang M, Han X, Liu H, Tian M, Ding C, Song J, Sun X, Liu Z, Yu S (2013) Inactivation of the $A B C$ transporter ATPase gene in Brucella abortus strain 2308 attenuated the virulence of the bacteria. Vet Microbiol 164:322-329

35. Pluschke G, Mayden J, Achtman M, Levine RP (1983) Role of the capsule and the $\mathrm{O}$ antigen in resistance of O18:K1 Escherichia coli to complementmediated killing. Infect Immun 42:907-913

36. Cross AS, Kim KS, Wright DC, Sadoff JC, Gemski P (1986) Role of lipopolysaccharide and capsule in the serum resistance of bacteremic strains of Escherichia coli. J Infect Dis 154:497-503

37. DeShazer D, Brett PJ, Woods DE (1998) The type II O-antigenic polysaccharide moiety of Burkholderia pseudomallei lipopolysaccharide is required for serum resistance and virulence. Mol Microbiol 30:1081-1100

38. Wang L, Wang Q, Reeves PR (2010) The variation of $O$ antigens in gramnegative bacteria. Subcell Biochem 53:123-152

39. Hackbarth CJ, Chambers HF (1993) blal and blaR1 regulate betalactamase and PBP 2a production in methicillin-resistant Staphylococcus aureus. Antimicrob Agents Chemother 37:1144-1149

40. Metcalf WW, Wanner BL (1993) Evidence for a fourteen-gene, phnC to phnP locus for phosphonate metabolism in Escherichia coli. Gene 129:27-32

41. O'Neil P, Lovell S, Mehzabeen N, Battaile K, Biswas I (2016) Crystal structure of histone-like protein from Streptococcus mutans refined to $1.9 \mathrm{~A}$ resolution. Acta Crystallogr F Struct Biol Commun 72:257-262

42. El-Sayed MM, Brown SR, Mupparapu K, Tolosa L (2016) The effect of pH on the glucose response of the glucose-galactose binding protein $\mathrm{L} 255 \mathrm{C}$ labeled with Acrylodan. Int J Biol Macromol 86:282-287

43. Ali L, Spiess M, Wobser D, Rodriguez M, Blum HE, Sakınç T (2016) Identification and functional characterization of the putative polysaccharide biosynthesis protein (CapD) of Enterococcus faecium U0317. Infect Genet Evol 37:215-224

44. Wang X, Xu X, Wu Y, Li L, Cao R, Cai X, Chen H (2013) Polysaccharide biosynthesis protein CapD is a novel pathogenicity-associated determinant of Haemophilus parasuis involved in serum-resistance ability. Vet Microbiol 164:184-189

45. Zhou H, Yang B, Xu F, Chen X, Wang J, Blackall PJ, Zhang P, Xia Y, Zhang J, Ma R (2010) Identification of putative virulence-associated genes of Haemophilus parasuis through suppression subtractive hybridization. Vet Microbiol 144:377-383

46. Whitfield C (2006) Biosynthesis and assembly of capsular polysaccharides in Escherichia coli. Annu Rev Biochem 75:39-68

47. Lin WS, Cunneen T, Lee CY (1994) Sequence analysis and molecular characterization of genes required for the biosynthesis of type 1 capsular polysaccharide in Staphylococcus aureus. J Bacteriol 176:7005-7016

\section{Submit your next manuscript to BioMed Central and we will help you at every step:}

- We accept pre-submission inquiries

- Our selector tool helps you to find the most relevant journal

- We provide round the clock customer support

- Convenient online submission

- Thorough peer review

- Inclusion in PubMed and all major indexing services

- Maximum visibility for your research

Submit your manuscript at www.biomedcentral.com/submit
(OioMed Central 